\title{
GENERALIZED 3-MANIFOLDS WHOSE NONMANIFOLD SET HAS NEIGHBORHOODS BOUNDED BY TORI
}

\author{
BY
}

MATTHEW G. BRIN ${ }^{1}$

\begin{abstract}
We show that all compact, ANR, generalized 3-manifolds whose nonmanifold set is 0-dimensional and has a neighborhood system bounded by tori are cell-like images of compact 3-manifolds if and only if the Poincare conjecture is true. We also discuss to what extent the assumption of the Poincare conjecture can be replaced by other hypotheses.
\end{abstract}

1. Introduction. In the search for a characterization of topological manifolds, the following arises.

Question 1. Is every ANR, generalized $n$-manifold ( $n$ finite) the cell-like image of a topological n-manifold?

Many results exist for $n \geqslant 5$. In this paper we give a partial result for $n=3$. Our result also gives a sufficient condition for an open 3-manifold to embed in a compact 3-manifold.

Since we restrict our discussion to ANR's (Absolute Neighborhood Retracts), we incorporate this into the following definition. A generalized $n$-manifold $\boldsymbol{M}$ is an $n$-dimensional retract of an open set of some finite dimensional Euclidean space $E^{k}$, with the following local homology property at every point $x$ in $M$ :

$$
H_{*}(M, M-\{x\}) \cong H_{*}\left(E^{n}, E^{n}-\{0\}\right) \text {. }
$$

The set of generalized $n$-manifolds properly contains the set of topological $n$-manifolds. Generalized manifolds arise as factors and cell-like images of topological manifolds. It is not known if all generalized manifolds are such.

A continuum $X$ in an ANR is cell-like if it contracts in any neighborhood of itself. A map from an ANR onto a separable metric space is cell-like if the inverse image of every point is cell-like.

Most recent results concerning Question 1 depend on the size of the nonmanifold set, the set of points each with no neighborhood homeomorphic to $E^{n}$. In [7] it is proven that every generalized $n$-manifold, $n \geqslant 5$, whose nonmanifold set has dimension less than $(n-1) / 2$ is the cell-like image of an $n$-manifold. A complete affirmative answer to Question 1 for $n \geqslant 5$ has been announced in [18].

Recent survey articles giving more information about the above topics (in various combinations) are [6], [13] and [19]. For more information about ANR's see [4].

Received by the editors March 27, 1979 and, in revised form, April 14, 1980.

AMS (MOS) subject classifications (1970). Primary 57A10, 54B15, 57A60, 57B05, 54C55, 55A40.

${ }^{1}$ Portions of this paper are contained in the author's doctoral dissertation written at the University of Wisconsin-Madison under the supervision of D. R. McMillan, Jr. 
McMillan has observed that for compact spaces, if $n=3$ and the nonmanifold set is 0-dimensional, then Question 1 is equivalent to:

Question 2. If $X$ is a compact generalized 3-manifold with 0-dimensional nonmanifold set, can the complement $M$ of the nonmanifold set be embedded in a compact 3-manifold $\bar{M}$ ?

Not every open $n$-manifold embeds in a compact $n$-manifold. In fact, there are open $n$-manifolds that are algebraically very simple (e.g., contractible) that embed in no compact $n$-manifold. In $[9, \S 5]$ it is shown that the contractible 3 -manifolds of [10] and [14] embed in no compact 3-manifold. Higher dimensional examples are found in [20].

To see the equivalence of Questions 1 and 2, we note that if such an embedding does exist, then a different $\bar{M}$ and embedding can be found so that the complement of $M$ in $\bar{M}$ is an intersection of handlebodies [5]. We can map $\bar{M}$ to $X$ using the identity on $M$ and mapping each component of $\bar{M}-M$ to the appropriate point of $X-M$. By [16, Theorem 3], each component of $\bar{M}-M$ will be a 1- $U V$ intersection of handlebodies and hence cell-like. (See below for a definition of 1-UV.)

If $X$ is a cell-like image under a map $f$ of a compact 3-manifold $\bar{M}^{\prime}$, then for each $x$ in the nonmanifold set of $X$, there is an open neighborhood $U$ of $f^{-1}(x)$ such that $U-f^{-1}(x)$ embeds in $E^{3}$ [11]. By [15] the restriction of $f$ to $U-f^{-1}(x)$ is cellular and by [1] is approximately a homeomorphism. Thus, a closed subset of $M$ together with a neighborhood of the inverse image of the nonmanifold set can be pieced together to get the desired $\bar{M}$. Some of these arguments require orientability near the nonmanifold set. This we have by the local homology properties of generalized manifolds.

Using the above observation we are able to prove the following.

THEOREM 1. Let Th be the set of compact generalized 3-manifolds satisfying

(i) the nonmanifold set is 0 -dimensional, and

(ii) every open neighborhood of the nonmanifold set contains a closed neighborhood of the nonmanifold set each component of which has connected torus boundary.

Then every $M$ in $\mathfrak{T}$ is the cell-like image of a compact 3-manifold if and only if the Poincaré conjecture is true.

Since property (ii) pervades this paper, we give it a name, saying that a space with this property has neighborhoods bounded by tori. A topological manifold has neighborhoods bounded by spheres. A result corresponding to Theorem 1 for spaces with neighborhoods bounded by spheres is found in [8]. In this case the conclusion is that every $M$ in $\mathscr{N}$ is a manifold if and only if the Poincare conjecture is true.

Theorem 1 follows directly from Theorem 2, which is stated and proved in the next section. Theorem 2 does not use the full hypothesis of Theorem 1. It is not known if this is a strict weakening of the hypotheses. Theorem 2 is stated as a criterion for embedding an open 3-manifold in a compact 3-manifold (compare with [23]).

The fact that, when $n=3$, the Poincare conjecture gets in the way of an affirmative answer to Question 1 is well known. The one point compactification of 
the locally finite connected sum of a countably infinite number of fake spheres is a generalized 3-manifold, but the manifold set (the complement of the nonmanifold set) embeds in no compact 3-manifold. In this example, the manifold set is not irreducible. In $\$ 3$ we construct an example showing that the Poincare conjecture is not avoided by assuming that the manifold set is irreducible.

Assumptions stronger than irreducibility do bear fruit. McMillan has shown, by methods different from ours, that if the manifold set $M$ is the ascending union of knot spaces (complements in $E^{3}$ of solid tori), then $M$ in fact embeds in $S^{3}$. Our methods reach the same conclusion under the stronger assumption that $M$ is an ascending union of solid tori. This last is stated as an addendum to Theorem 2.

The example referred to above and the example in $\$ 3$ are both one-point compactifications of open 3-manifolds. In general, such objects are not very friendly. We point out that if $M$ is the one-point compactification of the connected sum of a countably infinite number of homology spheres, then $H_{1}(M)$ is uncountably generated.

We will finish this section by setting down some definitions and conventions. In this paper, all manifolds and maps of manifolds will be in the PL category. An open manifold is noncompact and without boundary; a closed manifold is compact and without boundary. A 3-manifold is prime if every separating 2-sphere bounds a 3 -cell; it is irreducible if every 2-sphere bounds a 3-cell. We will use the symbols $I^{n}$, $S^{n}$ and $E^{n}$ to represent the $n$-dimensional ball, sphere and Euclidean space respectively. A torus (annulus, solid torus) is a space homeomorphic to $S^{1} \times S^{1}$ $\left(I \times S^{1}, I^{2} \times S^{1}\right)$. We will use $\mathrm{Bd}, \mathrm{Cl}$ and Int to denote boundary, closure and interior.

In a solid torus, $I^{2} \times S^{1}$, a curve embedded in the boundary in the same way as Bd $I^{2} \times\{p\}$ will be called a meridian, and a disk embedded in the solid torus in the same way as $I^{2} \times\{p\}$ will be called a meridional disk. If $M$ is an $n$-manifold with boundary, we say that we are adding $a k$-handle to $M$ by attaching the $n$-cell $I^{n-k} \times I^{k}$ to $\mathrm{Bd} M$ by a homeomorphism defined on $I^{n-k} \times \mathrm{Bd} I^{k}$. A $k$-cell intersecting a $k$-handle is consistent with the product structure if intersects in the set $\{p\} \times I^{k}$ for some $p$ in Int $I^{n-k}$.

We use $\operatorname{Link}(J, K)$ to denote the linking number of two disjoint loops $J$ and $K$. See [2, pp. 480-482] for a geometric description of linking over $Z_{2}$. The discussion there works perfectly well over $Z$.

For basic facts about incompressible surfaces, see [22, \$1.1]. Generally, the operation of compression, removing an essential annulus from the interior of a surface and sewing on a pair of disks to the newly created boundary components, is done on a surface embedded in a 3-manifold. In this paper, the term will also refer to performing the same operation on the domain of a singular map from a 2-manifold into a 3-manifold. Throughout this paper, the word surface should be taken to mean a singular map from a 2-manifold unless it is specified as nonsingular.

Theorem 2 refers to the Freudenthal compactification (also called the Ideal compactification). This can be defined as follows. Let $X$ be $\sigma$-compact and let $K_{1} \subset K_{2} \subset K_{3} \subset \ldots$ be a sequence of compact sets so that $X=\cup K_{i}$. An end of 
$X$ is a sequence $E=\left\{U_{1} \supset U_{2} \supset \ldots\right\}$ of nonempty, connected complementary domains of the sets $K_{i}$. The Freudenthal compactification of $X$ is $X$ with all "points" $E$ as above, a neighborhood base for $E$ being $\left\{U_{1}, U_{2}, \ldots\right\}$. Note that if $M$ is a compact manifold and $C$ is a closed, nonseparating, 0 -dimensional set in $M$, then the Freudenthal compactification of $M-C$ is $M$.

We list some local properties. A space is locally contractible at $p$ if every open set $U$ containing $p$ contains an open set $V$ containing $p$ so that $V$ contracts in $U$. A space is $k-L C$ at $p$ if every open $U$ about $p$ contains an open $V$ about $p$ so that every map of $S^{k}$ into $V$ extends to a map of $I^{k+1}$ into $U$. A set $X$ in a space $Y$ is $k-U V$ if every open $U$ containing $X$ contains an open $V$ containing $X$ so that every map of $S^{k}$ into $V$ extends to a map of $I^{k+1}$ into $U$. An open manifold $M$ will be said to be 1-acyclic at infinity if every compact set $K$ in $M$ is contained in a compact set $K^{\prime}$ so that every 1-cycle in $M-K^{\prime}$ bounds a 2-chain in $M-K$. Lastly, we will say that a set $X$ is simply connected $\bmod A \subset X$ if every loop in $X$ is homotopic to a product of conjugates in $X$ of loops in $A$.

\section{An embedding criterion.}

TheOREM 2. Let $\mathfrak{T}$ be the class of all connected, open 3-manifolds $M$ without boundary satisfying

(i) $M=\cup^{\infty} K_{i}$ where each $K_{i}$ is a connected, compact 3-manifold and $K_{i} \subset$ Int $K_{i+1}$.

(ii) $\mathrm{Bd} K_{i}$ is a union of tori, for all $i$.

(iii) Each component of $\mathrm{Bd} K_{i}$ separates $M$.

(iv) $M$ is 1-acyclic at infinity and

(v) $\hat{M}$, the Freudenthal compactification of $M$, is $1-L C$.

Then every $M$ in $\mathfrak{N}$ embeds in a compact 3-manifold if and only if the Poincaré conjecture is true.

ADDENDUM 2.1. If, in Theorem 2, hypothesis (ii) is replaced by (ii')

$K_{i} \cong I^{2} \times S^{1}$, then every $M$ in $\mathfrak{R}$ embeds in $S^{3}$ and the assumption of the Poincaré conjecture is unnecessary.

The two statements above will be proven together. The next lemma shows that property (iv) arises fairly naturally. The proof that follows, due to McMillan, is shorter than our original proof.

Lemma 2.2. If $M$ is a connected open 3-manifold and $H_{1}(M)=0$, then $M$ is 1-acyclic at infinity.

Proof. It suffices to show that every compact submanifold $K$ of $M$ is contained in a compact set $K^{\prime}$ of $M$ so that loops in $M-K^{\prime}$ bound in $M-K$. Since $K$ is compact, it has a finite number of boundary components. Each boundary component $F$ contains a bouquet of a finite number of loops $L_{F}$ so that $F-L_{F}$ is an open disk. Since $H_{1}(M)=0$, all the loops in all of the $L_{F}$ bound a finite number of surfaces in $M$ whose union $S$ is compact. Let $K^{\prime}$ be the compact set $K \cup S$. Let $J$ 
be a loop in $M-(K \cup S)$. Since $H_{1}(M)=0$, linking numbers for pairs of loops are well defined and symmetric. Since $J$ misses $S$, the linking number of $J$ and any loop in $L_{F}$ is zero. Thus, $J$ bounds a surface disjoint from the $L_{F}$. If this surface hits $K$ at all, it enters through open disks in Bd $K$. This surface can then be cut off on Bd $K$.

Proof of Theorem 2 and Addendum 2.1. The necessity of the Poincare conjecture is covered by the example of $\S 3$.

We establish some notation. Let $N_{i}=M-$ Int $K_{i}$ and $\hat{N}_{i}=\hat{M}-\operatorname{Int} K_{i}$. The $\hat{N}_{i}$ are the neighborhoods of the nonmanifold set with tori boundary referred to in Theorem 1. Note that hypotheses (ii) and (iii) of Theorem 2 require that each component of these neighborhoods have connected boundary.

We now construct the embedding of $M$ in a compact manifold $\bar{M}$. We first wish to enlarge the "layers" between the successive $K_{i}$ to obtain various properties. By choosing a subsequence of the $K_{i}$ and renumbering from 1, the 1-LC property lets us assume that all curves in $\hat{N}_{i}$ shrink in $\hat{N}_{i-1}, i \geqslant 2$. We choose yet a further subsequence.

The boundary of $K_{3}$ is compact, so $H_{1}\left(\mathrm{Bd} K_{3}\right)$ is generated by a finite number of 1-cycles. These bound a finite number of surfaces in $N_{2}$ which are contained in some $K_{j_{1}}$. Similarly surfaces bounded by generators of $H_{1}\left(\mathrm{Bd} K_{j_{1}+1}\right)$ in $N_{j_{1}}$ lie in some $K_{j_{2}}$. Continuing this way we get a subsequence $K_{2}, K_{3}, K_{j_{1}}, K_{j_{1}+1}, K_{j_{2}} \ldots$ which, if renumbered from 1, has the property that (i) loops in $N_{1}$ bound in $M$ and (ii) for every even number $i$, loops in Bd $K_{i}$ bound surfaces in $K_{i+1}-K_{i-1}$. We will work with this sequence through the remainder of the proof.

The embedding of $M$ in $\bar{M}$ will proceed by successively embedding the layers between the odd numbered $K_{i}$. To describe these embeddings, we need certain canonical curves on the components of $\mathrm{Bd} K_{i}$ for all odd $i \geqslant 3$. To keep the notation simple, we will describe these curves for $i=3$, the process for obtaining them for higher indexes being identical.

Let $Y=\mathrm{Cl}\left(K_{5}-K_{1}\right)$. It is most probable that $Y$ is not connected. We show that $Y$ embeds in a 3-manifold $Z$ (not necessarily connected) with $H_{1}(Z)=0$. We will use [21, Theorem 3.1] which says this can be done if $Y$ is orientable, $H_{1}(Y)$ is free and $i_{*}: H_{1}(\mathrm{Bd} Y) \rightarrow H_{1}(Y)$ is onto. Since loops in $N_{1}$ bound in $M$ we get immediately that $Y$ is orientable and that $i_{*}: H_{1}(\mathrm{Bd} Y) \rightarrow H_{1}(Y)$ is onto. From the long exact sequence for $(Y, \mathrm{Bd} Y)$ with reduced homology, $H_{1}(Y, \mathrm{Bd} Y) \cong$ $\tilde{H}_{0}(\mathrm{Bd} Y)$ which is free. By Lefschetz duality, $H^{2}(Y)$ is free, and by the Universal Coefficient Theorem $H_{1}(Y)$ is free.

We will regard $Y$ as a submanifold of both $M$ and $Z$. We will refer to $Z$ several times throughout the rest of the proof. Let $G$ be a component of $\operatorname{Bd} K_{3}$. Since $H_{1}(Z)=0, G$ separates $Z$. By hypothesis, $K_{3}$ is connected and $G$ separates $M$, so that if $U_{G}$ and $V_{G}$ are the complementary domains of $G$ in $Z$, one of them, say $V_{G}$, does not intersect $K_{3}-K_{1}$. By [21, Theorem 2.1], there is a pair of disjoint simple closed curves $A_{G}$ and $B_{G}$ on $G$ that intersect in a single point, transversely, and so that $B_{G}$ is null homologous in $\mathrm{Cl}\left(V_{G}\right)$ and $A_{G}$ is null homologous in $\mathrm{Cl}\left(U_{G}\right)$. We can find such curves on all components of $\mathrm{Bd} K_{3}$. 
The theorem will follow from:

Claim 1. Embed $K_{2 i+1}$ in a compact manifold $\bar{M}$ by attaching solid tori to the components $G_{j}$ of $\mathrm{Bd} K_{2 i+1}$ so that the curves $B_{G_{j}}$ are meridians for the solid tori. Then $K_{2 i+3}$ can be embedded in $\bar{M}$ so that $\mathrm{Cl}\left(\vec{M}-K_{2 i+3}\right)$ is a union of solid tori whose meridians are the curves $B_{G}$ for components of $\mathrm{Bd} K_{2 i+3}$ and so that the two embeddings agree on $K_{2 i-1}$.

Again, to keep the notation simple, we will show how an embedding for $K_{3}$ implies an embedding of $K_{5}$ so that the two embeddings agree on $K_{1}$.

First some homology preliminaries. Let $G$ be a component of Bd $K_{3}$. $G$ separates $Y$ into two sets, $Q=V_{G} \cap Y$ and $R=U_{G} \cap Y$. We know $B_{G}$ bounds a singular surface in $V_{G}$. This surface can be "cut off" on Bd $K_{4}$. Since curves on $\mathrm{Bd} K_{4}$ are null homologous in $K_{5}-K_{3}$, we can find a surface bounded by $B_{G}$ in $Q$. Similarly, $A_{G}$ bounds a surface in $U_{G}$. This surface may hit both $\mathrm{Bd} K_{2}$ and $\mathrm{Bd} K_{4}$. In the same manner, we get that $A_{G}$ bounds a surface in $R$. Note that while we have shown that $B_{G}$ is null homologous in $K_{5}-K_{3}$, we have not shown that $A_{G}$ is null homologous in $K_{3}-K_{1}$. We only know that $A_{G}$ is null homologous in the complementary domain of $G$ in $Y$ that intersects $K_{3}-K_{1}$.

This operation of "cutting off" a surface on an even numbered $\mathrm{Bd} K_{i}$ and redefining the surface in a more restricted area will be done several times during the proof.

Note that as defined above, $Q$ is a component of $K_{5}-K_{3}$. We now state:

Claim 2. Let $Q$ be a component of $K_{2 i+3}-K_{2 i+1}$. Let $\left\{G_{1}, \ldots, G_{n}\right\}$ be the components of $\mathrm{Bd} Q$ that are in $\mathrm{Bd} K_{2 i+3}$. Then each $A_{G_{i}}$ is homotopic in $Q$ to a product of the curves $\left\{B_{G_{j}}\right\}$.

We will finish the proof of Claim 1 before giving the proof of Claim 2. Let $K_{3}$ be embedded in $\bar{M}$ as given in the statement of Claim 1. Let $Q$ be a component of $\mathrm{Cl}\left(K_{5}-K_{3}\right)$. One boundary component $F_{Q}$ of $Q$ comes from Bd $K_{3}$. The other boundary components $\left\{G_{1}, \ldots, G_{n}\right\}$ of $Q$ come from Bd $K_{5}$. Form the space $\bar{Q}$ by sewing solid tori to the $\left\{G_{j}\right\}$ so that meridians are sewn to the curves $\left\{B_{G_{j}}\right\}$. The proof would be finished if $\bar{Q}$ were a solid torus with meridian $B_{F_{Q}}$. Such need not be the case however. Note that each component $F$ of $\mathrm{Bd} K_{3}$ has a component of $K_{5}-K_{3}$ as one of its complementary domains. We shall call its closure $Q_{F}$.

Each $\bar{Q}$ can be regarded as a submanifold of $\bar{K}_{5}$ obtained by sewing solid tori to all components of $\mathrm{Bd} K_{5}$ so that meridians are sewn to all the $B$ curves. Since every loop in $\hat{N}_{3}$ bounds a singular disk in $\hat{N}_{1}$, Claim 2 says that every loop in $\bar{K}_{5}-K_{3}$ bounds a singular disk in $\bar{K}_{5}-K_{1}$. Thus, all the components of Bd $K_{3}$ compress to embedded 2-spheres in $\bar{K}_{5}-K_{1}$.

Let $D_{1}, \ldots, D_{m}$ be a sequence of embedded disks in $\bar{K}_{5}-K_{1}$ along which all the components of $\mathrm{Bd} K_{3}$ compress. The compressions are done one at a time, starting with $D_{1}$. We can choose each Int $D_{i}$ disjoint from all components of $\mathrm{Bd} K_{3}$ as they exist after the first $i-1$ compressions. Since each component of $\mathrm{Bd} K_{3}$ is a torus, there are as many disks $D_{i}$ as components of $\mathrm{Bd} K_{3}$. We claim that each Bd $D_{i}$ can be chosen either an $A$ or $B$ curve for the component of $\mathrm{Bd} K_{3}$ that $D_{i}$ compresses. 
Let $F$ be a component of $\mathrm{Bd} K_{3}$. If $F$ compresses along a $D_{i}$ in $\overline{Q_{F}}$, then by cutting off $D_{i}$ on $\mathrm{Bd} K_{4}$ we get $\mathrm{Bd} D_{i}$ zero homologous in $Q_{F}$. Thus, $\operatorname{Bd} D_{i}$ is homotopic to $B_{F}$ on $F$ and $F$ compresses along $B_{F}$. If $D_{i}$ is not contained in $\overline{Q_{F}}$ it does not hit $Q_{F}$ and can be cut off on Bd $K_{2}$ and Bd $K_{4}$ ultimately showing, for reasons of homology, that $\mathrm{Bd} D_{i}$ is homotopic to $A_{F}$ on $F$.

Each time a component $F$ is compressed along a disk in $\overline{Q_{F}}$, a 1-handle is removed from $\overline{Q_{F}}$ and a 2 -handle is added to its complement. Other disks $D_{i}$ may run through this 2 -handle. We can require that the intersection is consistent with the product structure of the 2-handle.

We now switch our attention to the uncompressed components of $\mathrm{Bd} K_{3}$ as they are embedded in $\bar{M}$. We can compress these components along a sequence of disks $E_{1}, \ldots, E_{m}$ that correspond to the disks $D_{1}, \ldots, D_{m}$ in the sense that, for each $i$, $E_{i} \cap K_{3}=D_{i} \cap K_{3}$. This can be done, if $D_{i}$ lies in some $\bar{Q}$, by letting $E_{i}$ be a meridian of the solid torus bounded, in $\bar{M}-K_{3}$, by the component of $\mathrm{Bd} K_{3}$ containing Bd $D_{i}$. Otherwise, define $E_{i} \cap K_{3}$ to be $D_{i} \cap K_{3}$ and fill in the holes of $E_{i}$ using meridians of solid tori whose boundaries were compressed by earlier disks $E_{j}$ in the sequence.

Let $K_{3}^{*}$ denote $K_{3}$ after the compressions along the disks $D_{i}$. The space $K_{3}^{*}$ is obtained from $K_{3}$ by removing 1-handles and adding 2-handles. Let $K_{3}^{* *}$ denote $K_{3}$ after the compressions along the disks $E_{i}$. The spaces $K_{3}^{*}$ and $K_{3}^{* *}$ are naturally homeomorphic. Each of the complementary domains of $K_{3}^{* *}$ in $\bar{M}$ is either a solid torus minus a 1-handle, thus a 3-cell, or a solid torus plus a 2-handle sewn along some $A_{F}$. But $A_{F}$ and $B_{F}$ intersect transversely in one point for each $F$. Thus, all the complementary domains are 3-cells. The complementary domains $\bar{Q}^{*}$ of $K_{3}^{*}$ in $\bar{K}_{5}$ are bounded by one 2 -sphere each, and all loops in $\bar{Q}^{*}$ shrink in $\bar{K}_{5}$. Thus, each $\bar{Q}^{*}$ is simply connected and by hypothesis a 3-cell. Claim 1 is completed by mapping the $\bar{Q}^{*}$ to the complementary domains of $K_{3}^{* *}$ in $\bar{M}$ and reversing the steps of the compression. Since all of the disks $D_{i}$ and $E_{i}$ missed $K_{1}$, the new embedding agrees with the old on $K_{1}$.

Under the hypotheses of Addendum 2.1, we can say that each $A_{F}$ is a meridian of a solid torus. Also, using an argument of [23], we can say that if an infinite number of $\mathrm{Bd} K_{i}$ compress along $A_{F}$ in $K_{i}-K_{i-2}, i$ odd, then $M$ is a union of 3-cells. Otherwise, after a certain point, all of the compressing referred to above takes place in the complementary domains $\bar{Q}_{i}$ which must then be solid tori. Thus, $\bar{M}$ and each $\bar{K}_{i}$ is the union of two solid tori with transverse meridians and is $S^{3}$.

Proof of Claim 2. The following proof works because $\pi_{1}$ and $H_{1}$ of a torus are naturally isomorphic. Before we start the proof we mention one item of background. In general, if $X$ and $Y$ are 3-manifolds joined along boundary $F$, and $L$ is a loop in $X$ which bounds a disk $D$ in $X \cup Y$, then little can be said about the intersections of $D$ and $F$. In our present setting a great deal can be said. Our first proof was a lengthy sequence of cuts and homotopies that simplified $D \cap F$. What came out were facts about certain subgroups that were true without referring to a fixed $D$. The ghost of $D$ remains and bits and pieces can be found in what follows. Again, to simplify the notation, we show that the $A$ curves in $\mathrm{Bd} K_{5}$ are homotopic in $K_{5}-K_{3}$ to products of conjugates of the $B$ curves in $\mathrm{Bd} K_{5}$. 
Referring again to the technique of cutting surfaces off on the even numbered Bd $K_{i}$, we point out that if any loop in Bd $K_{5}$ bounds a disk in $K_{5}-K_{3}$, it must be a multiple of some $A$ curve, and if any loop in Bd $K_{5}$ bounds a disk in $\hat{N}_{5}$, it must be a multiple of a $B$ curve.

We will need some basic facts about the homological behavior of the $A$ and $B$ curves. Assume a multiple of an $A$ curve on Bd $K_{5}$ is zero homologous in $\hat{N}_{5}$. The surface it bounds can be cut off on $\mathrm{Bd} K_{6}$ and we would have a multiple of $A$ bounding in $K_{7}-K_{5}$. We know the $B$ curve that is transverse to $A$ bounds, for the same reason, in $K_{7}-K_{5}$. But two curves on the boundary of a 3-manifold with nonzero intersection number cannot both be torsion in $H_{1}$ of the 3-manifold. Thus, no multiple of $A$ bounds in $\hat{N}_{5}$.

We now wish to show that for each component $G$ of $\mathrm{Bd} K_{5}, A_{G}$ is homologous in $K_{5}-K_{3}$ to combinations of all the $B$ curves except $B_{G}$ in $\mathrm{Bd} K_{5}$. We will also show that the $B$ curves in $\mathrm{Bd} K_{5}$ are independent in $H_{1}\left(K_{5}-K_{3}\right)$. The second fact follows from the first since if there were a dependence relation, the free rank of the image of $i_{*}: H_{1}\left(\mathrm{Bd} K_{5}\right) \rightarrow H_{1}\left(K_{5}\right)$ would be less than the genus of $\mathrm{Bd} K_{5}$. This would violate a standard consequence of duality in 3-manifolds. To show the first statement, we again use the manifold $Z$ with $H_{1}(Z)=0$ in which $K_{5}-K_{1}$ embeds. Form a manifold $\bar{Z}$ by removing, for each component $G$ of Bd $K_{5}$, the complementary domain $V_{G}$ of $G$ in $Z$ that misses $K_{5}$, and replacing it with a solid torus $H_{G}$ so that a meridian of $H_{G}$ is sewn to $B_{G}$. According to [21, Theorem 2.1], each $B_{G}$ generates $H_{1}\left(U_{G}\right)$ where $U_{G}$ is the complementary domain of $G$ in $Z$ intersecting $K_{5}$. Thus, by replacing the domains $V_{G}$ one by one, we see that $H_{1}(\bar{Z})=0$. If we let $\bar{U}_{G}$ denote the complementary domain of $G$ in $\bar{Z}$ that intersects $K_{5}$, then each $A_{G}$ is zero homologous in $\bar{U}_{G}$. If not, some other curve $L_{G}$ is by [21, Theorem 2.1] since $H_{1}(\bar{Z})=0$. The surface bounded by $L_{G}$ can be adjusted to hit all the solid tori $H_{G_{i}}$ in meridinal disks. The surface can also be cut off on $\mathrm{Bd} K_{4}$. Note that the only intersection of this surface with $G$ is $L_{G}$. Thus, $L_{G}$ is homologous in $K_{5}-K_{3}$ to a combination of $B$ curves in $\mathrm{Bd} K_{5}$ other than $B_{G}$. But, each $B_{G_{j}}$ is zero homologous in $V_{G_{j}}$ in $Z$. Thus, each $L_{G}$ is zero homologous in $U_{G}$ in $Z$ and must be homotopic to $A_{G}$ on $G$.

We now derive some properties of $H_{1}\left(\mathrm{Bd} K_{5}\right)$ that follow from the fact that $\hat{M}$ is 1-LC. Let $H$ denote $H_{1}\left(\mathrm{Bd} K_{5}\right)$. There is a natural isomorphism between $H$ and the direct sum of the fundamental groups of the components of $\mathrm{Bd} K_{5}$. We will define a sequence of subgroups of $H, 0 \subset S_{1} \subset S_{2} \subset \cdots \subset S_{n}=H$. We let $S_{1}$ be the subgroup of $H$ generated by all loops in Bd $K_{5}$ that are null homotopic in $\hat{N}_{5}$. Let $S_{2}$ be the subgroup generated by loops in Bd $K_{5}$ that are homotopic in $K_{5}-K_{3}$ to products of conjugates of loops in $S_{1}$. The group $S_{3}$ will be loops homotopic in $\hat{N}_{5}$ to products of conjugates of loops in $S_{2}$. We continue alternating in this fashion. Even numbered groups are loops that are null homotopic in $K_{5}-K_{3}$ mod loops in lower groups and odd numbered groups are defined similarly via $\hat{N}_{5}$. Since $H$ is finitely generated and since every loop in $\mathrm{Bd} K_{5}$ bounds a disk in $\hat{N}_{3}$, there is a finite $n$ such that $S_{n}=H$. Choose $n$ to be the smallest with this property. For $1 \leqslant i<j \leqslant n$ we cannot have $S_{i}=S_{j}$. 
We now analyze the structure of the groups $S_{i}$. We can represent $H$ as a direct sum $H_{A} \oplus H_{B}$ where $H_{A}$ is the subgroup of $H$ generated by $A$ curves in $\mathrm{Bd} K_{5}$ and $H_{B}$ is the subgroup generated by the $B$ curves in $\mathrm{Bd} K_{5}$. By the homology properties of the $A$ and $B$ curves, $S_{1}$ is a subgroup of $H_{B}$; call it $B_{1}$.

We claim there are sequences of subgroups $B_{1} \subset B_{3} \subset B_{5} \subset \cdots \subset H_{B}$ and $A_{2} \subset A_{4} \subset A_{6} \subset \cdots \subset H_{A}$ so that for odd $i, S_{i}=B_{i} \oplus A_{i-1}$ and for even $i$, $S_{i}=A_{i} \oplus B_{i-1}$. We further claim that for even $i$, each loop in $A_{i}$ is null homotopic in $K_{5}-K_{3}$ mod loops in $B_{i-1}$. This last statement completes the proof of Claim 2. It will also be true that for odd $i$, each loop in $B_{i}$ is null homotopic in $\hat{N}_{5}$ mod loops in $A_{i-1}$.

The proof of these claims proceeds by induction. We will show the inductive step for even $i$. The inductive step for odd $i$ is similar and simpler since each component of $\hat{N}_{5}$ has connected boundary.

We consider $S_{2 k}$. Let $L$ be a loop in $S_{2 k}$. $L$ lies on some component $G$ of Bd $K_{5}$ and can be represented as $a A_{G}+b B_{G}$. We claim that $b B_{G}$ is in $B_{2 k-1}$. Since $L$ is in $S_{2 k}$, there is a genus zero surface $C_{1}$ in $K_{5}-K_{3}$, one of whose boundary components is $L$ and which has all other boundary components in $S_{2 k-1}=B_{2 k-1} \oplus$ $A_{2 k-2}$. By the inductive hypotheses, each loop in $A_{2 k-2}$ bounds a genus zero surface in $K_{5}-K_{3}$ together with loops in $B_{2 k-3} \subset B_{2 k-1}$. Thus, $C_{1}$ can be replaced by a genus zero surface $C_{2}$ in $K_{5}-K_{3}$ with $L$ as one boundary component and loops in $B_{2 k-1}$ for all other boundary components. However, $a A_{G}$ together with multiples of $B$ curves from components of $\mathrm{Bd} K_{5}$ other than $G$ bounds a 2-chain $C_{3}$ in $K_{5}-K_{3}$. Thus, $C_{2}-C_{3}$ is a 2-chain in $K_{5}-K_{3}$ whose boundary lies in $H_{B}$. But, the $B$ curves are independent in $H_{1}\left(K_{5}-K_{3}\right)$, so the coefficient of each $B$ in the boundary of $C_{2}-C_{3}$ is zero. But $\operatorname{Bd}\left(C_{2}-C_{3}\right) \cap G$ is $\left(\operatorname{Bd} C_{2} \cap G\right)-a A_{G}$ since $C_{3}$ hits $G$ only in $a A_{G}$. The boundary of $C_{2}$ on $G$ is $a A_{G}+b B_{G}+$ elements in $B_{2 k-1}$. So $\operatorname{Bd}\left(C_{2}-C_{3}\right) \cap G$ is $b B_{G}+$ elements in $B_{2 k-1}$. Thus, $b B_{G}$ is in $B_{2 k-1}$. Now the genus zero surface $C_{2}$ shows that $a A_{G}$ is null homotopic in $K_{5}-K_{3} \bmod$ loops in $B_{2 k-1}$ and the proof of Claim 2 is complete.

3. If the Poincare conjecture is false. We start with a description of the example. Let $P$ be a prime homotopy 3-sphere that is not homeomorphic to $S^{3}$. Let $C$ be a 3-cell in $P$ containing a spanning arc $A$ and a simple, closed curve $J_{1}$ arranged as in Figure 1. $J_{1}$ bounds an embedded disk in $C$ but not in $C-A$.

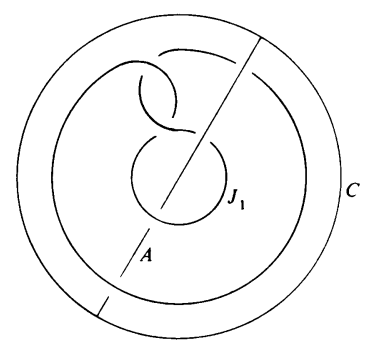

Figure 1 
Let $J_{2}$ be a simple closed curve in $P$ contained in no 3-cell (see [3]) so that $J_{2} \cap C=A$. Let $T_{i}$ be a solid torus regular neighborhood of $J_{i}, i=1,2$. Let $F=\operatorname{Bd} T_{1}$ and $G=\operatorname{Bd} T_{2}$.

Let $Q=\mathrm{Cl}\left(P-T_{1}-T_{2}\right)$. The boundary of $Q$ is $F \cup G$.

We have $\pi_{1}\left(P-J_{1}\right)=\pi_{1}(P-C) * \pi_{1}\left(C-J_{1}\right)=\{1\} * Z=Z$. Since $\operatorname{Link}\left(J_{1}, J_{2}\right)=0$, the curve $J_{2}$ must be homotopically trivial in $\left(P-J_{1}\right)$. There is, therefore, a simple closed curve $Y$ in $G$ that bounds a surface in $Q$. $Y$ is parallel to $J_{2}$ in $T_{2}$ and transverse to a meridian $Z$ of $T_{2}$ (see Figure 2).

The curve $J_{1}$ bounds a surface in $P-T_{2}$, so there is a curve $X$ in $F$, parallel to $J_{1}$ in $T_{1}$, that bounds a surface in $Q . X$ is transverse to a meridian $W$ of $T_{1}$. The curve $X$ and two parallel copies of $Z$ bound an embedded disk with two holes in $C-$ Int $T_{2}$ (see Figure 2).

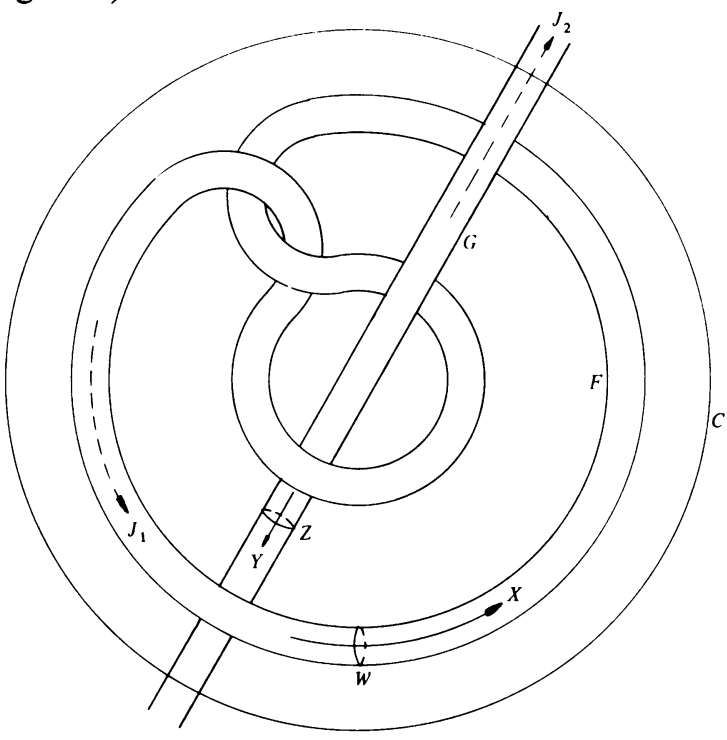

FIGURE 2

Let $Q_{1}, Q_{2}, \ldots$ be disjoint spaces homeomorphic to $Q$ with $W_{i}, X_{i}, Y_{i}, Z_{i}, F_{i}$ and $G_{i}$ in $Q_{i}$ corresponding to $W, X, Y, Z, F$ and $G$ in $Q$ respectively. Let $M=T \cup Q_{1}$ $\cup Q_{2} \cup \ldots$ with $Q_{i}$ sewn to $Q_{i+1}$ by identifying $\left(G_{i}, Y_{i}, Z_{i}\right)$ to $\left(F_{i+1}, W_{i+1}, X_{i+1}\right)$, and with $T$ a solid torus whose boundary is sewn to $F_{1}$ so that a meridian is sewn to $W_{1}$.

Let $\hat{M}$ be the 1-point compactification of $M$.

THEOREM 3. The space $\hat{M}$ is a compact generalized 3-manifold such that

(i) the nonmanifold set consists of one point with neighborhoods bounded by tori,

(ii) the manifold set is irreducible, and

(iii) the manifold set embeds in no compact 3-manifold.

Proof. First some notation. Let $p$ be the point in $\hat{M}-M$. We will use $K_{n}$ to denote $T \cup Q_{1} \cup \cdots \cup Q_{n-1}$. We will use $N_{n}$ to denote $\mathrm{Cl}\left(M-K_{n}\right)$ and $\hat{N}_{n}$ to denote $\mathrm{Cl}\left(\hat{M}-K_{n}\right)$. Note that $Q_{n}$ is contained in $N_{n}$ and $\hat{N}_{n}$ but not in $K_{n}$. The 
proof is divided into a number of steps. The first few steps, and the bulk of the proof, concern themselves with the local contractibility of $\hat{M}$. The remaining steps cover the local homology properties. The primary reasons that $\hat{M}$ is locally contractible seem to be that $P-J_{1}$ has trivial $\pi_{2}$ and that each $\operatorname{Bd} \hat{N}_{i}$ bounds a finite 3-chain in $\hat{N}_{i}$. These combine to give the local 2-connectedness, the major stumbling block.

Step 1, a preliminary, proves item (ii). After that our attention focuses on the point $p$, the only point in the nonmanifold set.

Step 1. The manifold set is irreducible. We show that $G$ is incompressible in $\mathrm{Cl}\left(P-T_{2}\right)$ and $F$ is incompressible in $Q$. If $G$ compresses, it must compress along $Y$, implying that $J_{2}$ is contained in a 3-cell, contradicting our choice of $J_{2}$. If $F$ compresses, it must compress along $X$, implying that $J_{1}$ bounds an embedded disk $D$ in $P-J_{2}$. If $L$ is a simple closed curve of $\operatorname{Bd} C \cap D$, then $L$ does not link $J_{2}$. Thus, one of the disks bounded by $L$ in $\operatorname{Bd} C$ misses $J_{2}$, and $D$ can be cut off on Bd $C$. This implies that $J_{1}$ bounds an embedded disk in $C-A$, contradicting our choice of $A$ and $J_{1}$ in $C$.

Since $J_{2}$ is contained in no 3 -cell and since $P$ is prime, $T \cup Q_{1}$ and all $Q_{i}, i \geqslant 2$, are irreducible. Also, $T \cup Q_{1}$ and all $Q_{i}, i \geqslant 2$, have incompressible boundary. Thus, every $K_{i}$ is irreducible and $M$ is irreducible.

Step 2. $\hat{M}$ is 1-LC at $P$. We observe first that every $Z_{i}$ bounds a disk in $\hat{N}_{i+1}$ since $Z_{i}$ is $X_{i+1}$, and $X_{i+1}$ and two copies of $Z_{i+1}$ bound a disk with two holes in $Q_{i+1}$. The holes can be partly filled in by disks with two holes in $Q_{i+2}$. Continuing in this way we fill in the disk by mapping a Cantor set to $p$.

Also note that $W_{i}$ (which equals $Y_{i-1}$ ) bounds a disk in $\hat{N}_{i-1}$. Temporarily regarding $Q_{i-1}$ as a subset of $P$ and recalling that $J_{2}$ was homotopic to zero in $P-J_{1}$, we see that $Y_{i-1}$ with several copies of $Z_{i-1}$ bounds a disk with holes in $Q_{i-1}$. These holes can be filled in with disks in $\hat{N}_{i}$ as above.

Now any loop in $Q_{i}$ will bound a disk with holes together with copies of $W_{i}$ and $Z_{i}$. Thus, any loop in $Q_{i}$ bounds a disk in $\hat{N}_{i-1}$. What follows is essentially an argument of Kozlowski.

Let $L$ be a loop in $\hat{N}_{i}$. By a small homotopy, put $L$ in general position with respect to all the $\mathrm{Bd} N_{j}$. By the previous paragraphs, there is a homotopy, contained in $\hat{N}_{i-1}$, taking each arc of $L \cap Q_{i}$ to an arc in $\mathrm{Bd} N_{i+1}$ keeping the endpoints fixed. Now $L$ lies in $\hat{N}_{i+1}$. Similarly a homotopy contained in $\hat{N}_{i}$ carries $L$ into $\hat{N}_{i+2}$. Continuing, we can put these homotopies together to define a homotopy on $[0,1)$. Since the homotopies near 1 can be kept arbitrarily close to $p$, we can extend the homotopy continuously to all of $[0,1]$ by making the 1 level the constant map to $p$.

Step 3. For each $i \geqslant 1$, there is a map $f_{i}: T \rightarrow \hat{N}_{i}$, where $T$ is a solid torus and $\left.f_{i}\right|_{\mathrm{Bd} T}: \mathrm{Bd} T \rightarrow \mathrm{Bd} \hat{N}_{i}$ is a homeomorphism.

This will be needed to show that $\hat{M}$ is 2 -LC at $p$. Since all the $\hat{N}_{i}$ are homeomorphic, we will construct the map $f_{1}$.

Divide $P$ into the 3 -cell $C$ and its complement $C^{\prime}$. The space $\mathrm{Cl}\left(C-T_{1}\right)$ is homeomorphic to the solid torus $T$ minus the interior of a 3-cell $B$ in Int $T$. There 
is a map from the 3-cell $B$ to $C^{\prime}$ taking the boundary homeomorphically to the boundary. Putting these together gives a map $f$ from $T$ to $\operatorname{Cl}\left(P-T_{1}\right)$ that takes the boundary homeomorphically to the boundary. We can put $f$ in general position with respect to $J_{2}$.

The set $f^{-1}\left(J_{2}\right)$ consists of a finite number of disjoint simple closed curves $L_{1}, \ldots, L_{n}$ with all but one, say $L_{1}$, contained in $B$. The curve $L_{1}$ intersects $B$ in a spanning arc, possible knotted. If $L_{1} \cap B$ is replaced by an unknotted spanning arc, creating a curve $L_{1}^{*}$, then the embedding of $L_{1}^{*}$ in $T$ is that of the standard Whitehead link (see Figure 3).

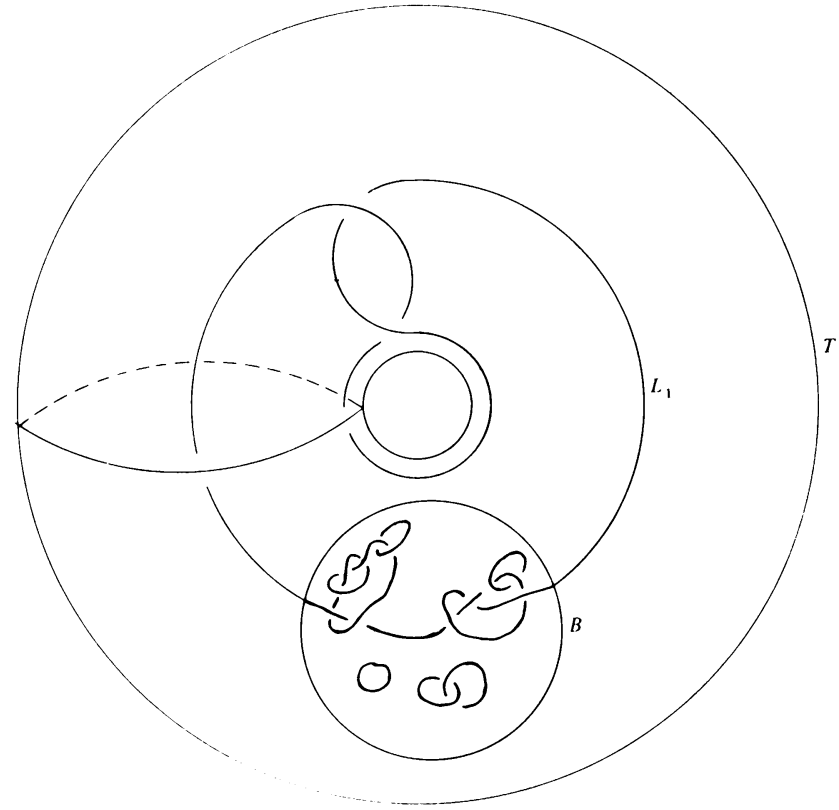

FIGURE 3

We can adjust $f$ by a homotopy on a small neighborhood of $f^{-1}\left(J_{2}\right)$ so that a neighborhood $T_{L}$ of $L_{1}$ is carried homeomorphically onto $T_{2}$ and so that $f^{-1}\left(T_{2}\right)$ is a regular neighborhood of $f^{-1}\left(J_{2}\right)$ one of whose components is $T_{L}$. The space $T-f^{-1}\left(T_{2}\right)$ is carried by $f$ onto $Q_{1}$. We wish to redefine $f$ on $f^{-1}\left(T_{2}\right)$ so the new map takes $f^{-1}\left(T_{2}\right)$ to $\hat{N}_{2}$. We will do this first on neighborhoods of $L_{i}$ for $i>1$.

Let $T_{i}$ be the component of $f^{-1}\left(T_{2}\right)$ that contains $L_{i}$. Note that $f$ carries $\operatorname{Bd} T_{i}$ into an annulus of $G_{1}$, the boundary component that $Q_{1}$ shares with $Q_{2}$. This annulus is a neighborhood of $Z_{1}$ on $G_{1}$. We can adjust $f$ by a homotopy so that $f$ carries Bd $T_{i}$ into $Z_{1}$. Since $Z_{1}$ bounds a disk in $\hat{N}_{2}$, we can extend the map to take $T_{i}$ to $\hat{N}_{2}$ by first extending the map to map $T_{i}$ into a 2 -disk bounded by $Z_{i}$ by the Tietze extension theorem and then mapping the disk into $\hat{N}_{2}$ with boundary $Z_{1}$.

This leaves the solid torus neighborhood of $L_{1}$. We can fill that in by mapping that solid torus minus a "knotted Whitehead link" into $\hat{N}_{2}$ in a manner identical to the above. Continuing this way gives a map defined on all of $T$ except on a certain cell-like continuum. Points close to that continuum are mapped close to $p$. The 
map $f_{1}$ is completed by mapping the continuum to $p$. Note that $f^{-1}(p)$ contains components other than this continuum, namely, simple curves essential in solid tori that are contained in 3-cells in $T$.

Step 4. $\hat{M}$ is 2-LC at p. Let $f: S^{2} \rightarrow \hat{N}_{i}$ be a singular map of a 2-sphere into $\hat{N}_{i}$. We wish to show that $f$ extends to a map of $I^{3}$ into $\hat{N}_{i-1}$ where $S^{2}$ is $\mathrm{Bd} I^{3}$. We will do this in pieces.

Put $f$ in general position with respect to the surfaces Bd $N_{j}$. Each simple closed curve $L$ of $f^{-1}\left(\mathrm{Bd} N_{j}\right)$, all $j$, bounds an embedded disk $D_{L}$ in $I^{3}$ with $D_{L} \cap S^{2}=$ $L$. Since all the curves $L$ are disjoint, we can choose these disks disjoint (there are a countable number). Fix an $L$ in $f^{-1}\left(\operatorname{Bd} N_{j}\right)$. We wish to extend $f$ to $D_{L}$ very explicitly. Since Bd $N_{j}$ is a torus with $\pi_{1}$ generated by $W_{j}$ and $X_{j}, f(L)$ is homotopic to a loop $L^{\prime}$ that follows $W_{j}$ around $n$ times and then $X_{j}$ around $m$ times, with $x$, the point $W_{j} \cap X_{j}$, used as a base point. We can homotop $f$ near $L$ so that $f$ takes $L$ to such a loop. Choose two points $x_{1}$ and $x_{2}$ in $f^{-1}(x)$ so that one complementary domain of $x_{1}$ and $x_{2}$ in $L$ goes to $n W_{j}$ and the other to $m X_{j}$.

Including $x_{2}$, there are $m+n-1$ points in $f^{-1}(x)$ other than $x_{1}$. Join these $m+n-1$ points to $x_{1}$ by arcs in $D_{L}$ that are disjoint except at $x_{1}$ and whose interiors lie in Int $D_{L}$. Extend the map $f$ to these arcs by defining it to be the constant map to $x$ on them. The disk $D_{L}$ has been carved into $m+n$ subdisks. Each of their boundaries maps either to $W_{j}$ or to $X_{j}$. We extend $f$ to these subdisks of $D_{L}$ exactly as specified in the first two paragraphs of Step 2.

We will now regard $f$ as being defined on $S^{2}$ and all the disks $D_{L}$. The disks $D_{L}$ carve $I^{3}$ into a countable number of 3-cells with $f$ defined on the boundary of each. Note that $f$ carries each $D_{L}$ only one neighborhood farther from $p$ than it carries Bd $D_{L}$. Thus, for all $j, f^{-1}\left(\hat{N}_{j}\right)$ contains the boundaries of all but a finite number of these 3-cells. We will be done if we can extend $f$ to each of these 3-cells so that $f$ does not carry the interior much farther from $p$ than it carries its boundary.

We now, therefore, reduce the problem and regard $I^{3}$ as one of the subcells whose boundary consists of a connected surface $S_{0}^{\prime}$ in the original 2-sphere plus a collection of disks $D_{L}$. The map $f$ takes $S_{0}^{\prime}$ into some $Q_{i}$ with the interior of $S_{0}^{\prime}$ carried into the interior of $Q_{i}$. The boundaries of the subdisks of each $D_{L}$ are carried to the curves $W_{i}, X_{i}, Y_{i}$ and $Z_{i}$. Recall that the disks constructed in Step 2 bounded by $X_{i}$ and $Y_{i}$ had neighborhoods of their boundaries mapped into $Q_{i}$. Thus, if we move $f$ by a small homotopy that pulls the images of the $\operatorname{arcs}$ in $D_{L}$ defined above into the interior of $Q_{i}$, we get a different description of $\mathrm{Bd} I^{3}$. We now have a genus zero surface $S_{0}$ in Bd $I^{3}$ obtained from $S_{0}^{\prime}$ by adding neighborhoods of those arcs in the disks $D_{L}$ that were part of the boundaries of subdisks whose boundaries went to $W_{i}$ or $Z_{i}$ under $f$. Also, added to $S_{0}^{\prime}$ are those parts in $Q_{i}$ of the subdisks of $D_{L}$ whose boundaries went to $X_{i}$ and $Y_{i}$ under $f$.

We now have $S_{0}$ mapped to $Q_{i}$ by $f, f\left(\mathrm{Bd} S_{0}\right) \subset \operatorname{Bd} Q_{i}, f\left(\operatorname{Int} S_{0}\right) \subset \operatorname{Int} Q_{i}$, and each boundary component of $S_{0}$ mapped to curves parallel to $W_{i}$ or $Z_{i}$. The disks of $\left(\mathrm{Bd} I^{3}\right)-S_{0}$ are the disks described in Step 2 bounded by curves parallel to $W_{i}$ and $Z_{i}$. Note that portions of $\mathrm{Bd} I^{3}$ other than $S_{0}$ are mapped into $Q_{i}$, namely parts of the disks constructed in Step 2 bounded by curves parallel to $W_{i}$. 
We would like to extend $\left.f\right|_{\mathrm{Bd}} I^{3}$ to all of $I^{3}$. We will do that by first constructing a map which takes a 3-cell into $\hat{N}_{i}$ and which takes the boundary of the 3-cell to a set somewhat different from $f\left(\mathrm{Bd} I^{3}\right)$. We will then show how to use this new map to extend $f$ to all of $I^{3}$.

Orient $Z_{i}, W_{i}$ and $S_{0}$. Orient the boundary components of $S_{0}$ consistently with $S_{0}$. Since $Z_{i}$ and $W_{i}$ are independent generators of $H_{1}\left(Q_{i}\right)=Z \oplus Z$, there must be as many boundary components of $S_{0}$ mapping to curves on $G_{i}$ parallel to $Z_{i}$ as antiparallel to $Z_{i}$. Similarly, for $W_{i}$ on $F_{i}$. Thus, we can add annuli between pairs of components of Bd $S_{0}$ that map to $G_{i}$ and send the annuli into $G_{i}$, and we can add annuli between pairs of components of $\mathrm{Bd} S_{0}$ that map to $F_{i}$ and send these annuli into $F_{i}$ to create a closed orientable singular surface $S_{1}$ that maps into $Q_{i}$.

We can regard $Q_{i}$ as a submanifold of $P$ with $S_{1}$ mapping into $\operatorname{Bd} T_{1}$ and $\operatorname{Bd} T_{2}$ along the annuli added in the last paragraph. We can remove the annuli mapping into Bd $T_{2}$ and cap off the holes with disks that map onto meridinal disks of $T_{2}$. This only partially reconstructs a sphere, leaving us with a surface that we will call $S_{2}$. We have to be more careful in dealing with $T_{1}$.

Let $A$ be an annulus added in the creation of $S_{1}$ that maps into Bd $T_{1}$. Let $E$ be an arc in $A$ joining its two boundary components. Let $E^{\prime}$ be an arc in $S_{0}$ connecting the endpoints of $E$. We can do this for all the annuli mapping into Bd $T_{1}$ keeping the arcs $E^{\prime}$ disjoint since $S_{0}$ is a sphere with holes.

Let $E \cup E^{\prime}$ be one of the simple closed curves on $S_{2}$ just constructed. Its image may not shrink in $P-\operatorname{Int} T_{1}$. However, $W$ generates $\pi_{1}\left(P-\operatorname{Int} T_{1}\right)$ and the annulus containing $E$ contains a curve $W^{\prime}$ that maps to a curve parallel to $W$ and that pierces $E$ once. Thus, $E$ can be replaced by another arc $E^{\prime \prime}$ in the annulus by adding multiples of $W^{\prime}$, so that the image of $E^{\prime \prime} \cup E^{\prime}$ bounds a singular disk in $P$ - Int $T_{1}$. Do this for each annulus.

We will now compress the surface $S_{2}$ by replacing annular neighborhoods of the curves $E^{\prime \prime} \cup E^{\prime}$ by pairs of disks that map into $P$ - Int $T_{1}$. This gives us a sphere $S_{3}$ that maps into $P$ - Int $T_{1}$. We can make sure that the images of the new disks hit $T_{2}$ in meridional disks.

Since $P$ is a homotopy 3 -sphere and $T_{1}$ is connected, the sphere theorem [17] says that $\pi_{2}\left(P-\right.$ Int $\left.T_{1}\right)=0$. Thus, $S_{3}$ bounds a 3-cell that maps into $P-\operatorname{Int} T_{1}$. We have already identified $Q_{i}$ with $\mathrm{Cl}\left(P-T_{1}-T_{2}\right)$. We can map $T_{2}$ to $\hat{N}_{i+1}$ by Step 3. Recall that this map was badly behaved only on certain 3-cells. We can insure that these 3-cells do not intersect the meridional disks in $T_{2}$ that are in the image of $S_{3}$.

Certain meridional disks of Image $\left(S_{3}\right) \cap T_{2}$ were meridional disks of Image $\left(S_{2}\right)$ $\cap T_{2}$. These will be carried onto the disks in $\hat{N}_{i+1}$ capping off those components of Bd $S_{0}$ mapped to curves parallel to $Z_{i}$.

We have carried $S_{3}$ and the 3-cell it bounds into $\hat{N}_{i}$. We wish to show that $f\left(\mathrm{Bd} I^{3}\right)$ bounds a 3-cell. Unfortunately, $S_{3}$ and $\mathrm{Bd} I^{3}$ do not coincide. We will analyze their differences. In what follows, certain letters will represent collections of objects.

To obtain $S_{3}$ from Bd $I^{3}$, pairs of disks $D$ whose boundaries map to curves 
parallel to $W_{i}$ are removed and replaced by annuli $A$. Annuli $\tilde{A}$ that are neighborhoods of the curves $E^{\prime \prime} \cup E^{\prime}$ are removed and replaced by pairs of disks $\tilde{D}$. The annuli $A$ and the annuli $\tilde{A}$ are paired, one from $A$ with one from $\tilde{A}$, with each associated pair intersecting transversely in a disk and all other combinations disjoint.

For each pair in $\tilde{D}$, the images of the two disks are parallel off $p$ since the map from $T_{2}$ to $\hat{N}_{i+1}$ is well behaved off certain 3-cells, and the parallel pairs can be kept close together in $T_{2}$ so that the 3-cells containing the bad part of the map of Step 3 do not get between them.

Thus, each annulus from $\tilde{A}$ with its associated pair of disks from $\tilde{D}$ bounds a 3-cell mapping into $\hat{N}_{i}$ which can be added to the 3-cell bounded by $S_{3}$ as a 1-handle. This removes the disks $\tilde{D}$ from $S_{3}$ and adds in the annuli $\tilde{A}$. This turns the 3-cell bounded by $S_{3}$ into a handlebody mapping into $\hat{N}_{i}$.

We now point out that the disk pairs $D$ map to parallel copies of the disks of Step 2 bounded by $W_{i}$. Each of these pairs together with the appropriate annulus from $A$ bounds a singular 3-cell mapping into $\hat{N}_{i-1}$. Each of these 3-cells can be added to the handlebody of the last paragraph as a 2-handle. By this process Bd $I^{3}$ is recovered. The 1-handles and 2-handles are transverse in pairs and cancel. Thus, a 3-cell that maps into $\hat{N}_{i-1}$ and is bounded by $\mathrm{Bd} I^{3}$ is created. There is a map from $I^{3}$ to this 3-cell that is the identity on Bd $I^{3}$. Step 4 is complete.

Step 5. $\hat{M}$ is locally contractible. Each $\hat{N}_{i}$ collapses to a subset of its 2-skeleton $\cup\{p\}$. We thank D. R. McMillan for pointing this out. One way to accomplish this is to take a triangulation of $N_{i}$ small with respect to the various $Q_{j}$ and start collapsing at $\mathrm{Bd} N_{i}$. We can demand that all 3-simplexes in a $Q_{j}$ be collapsed before starting on any in $Q_{j+2}$. Thus, points near $p$ will not be moved far from $p$ in the process. This much care is not really needed since each $Q_{j}$ is compact and there are an infinite number of $\hat{N}_{k}$.

It is now a standard exercise to pull the various skeleta near $p$ into $p$ within a slightly larger neighborhood.

We now look at the local homology properties of $\hat{M}$. By excision it is sufficient to show $H_{*}\left(\hat{N}_{i}, N_{i}\right) \cong H_{*}\left(E^{3}, E^{3}-\{0\}\right)$ for one $i$. We will consider the following exact sequence:

$$
\begin{aligned}
H_{3}\left(\hat{N}_{i}\right) & \rightarrow H_{3}\left(\hat{N}_{i}, N_{i}\right) \rightarrow H_{2}\left(N_{i}\right) \rightarrow H_{2}\left(\hat{N}_{i}\right) \rightarrow H_{2}\left(\hat{N}_{i}, N_{i}\right) \\
& \rightarrow H_{1}\left(N_{i}\right) \rightarrow H_{1}\left(\hat{N}_{i}\right) \rightarrow H_{1}\left(\hat{N}_{i}, N_{i}\right) \rightarrow 0 .
\end{aligned}
$$

We will use the following observation. Since each $\hat{N}_{j}$ contracts in some $\hat{N}_{j-k}(k$ fixed), then each $n$-cycle in $\hat{N}_{j}$ is homologous to an $n$-cycle in $\operatorname{Bd} \hat{N}_{j}$. This is geometrically obvious and can be shown using excision.

Step 6. The groups $H_{3}\left(\hat{N}_{i}\right), H_{2}\left(\hat{N}_{i}\right)$ and $H_{1}(M)$ are trivial and $H_{2}\left(N_{i}\right) \cong Z$. The first two we get from the above observation, from the fact that $H_{3}\left(\operatorname{Bd} \hat{N}_{i}\right)=0$ and from the map of Step 3. We get $H_{1}(M)=0$ since every loop in $Q_{i}$ bounds $\bmod W_{i}$ and $Z_{i}$ which each bound in $Q_{i-1}$ and $Q_{i+1}$ respectively. Since $H_{1}(M)=0$, every surface in $M$ separates. Since $M$ has one end, every surface bounds. We can show $H_{2}\left(N_{i}\right) \cong Z$ by the Mayer-Vietoris sequence. 
Step 7. The map $i_{*}$ is an isomorphism. Local simple connectivity shows that $i_{*}$ is surjective. Loops passing through $p$ can be pulled slightly off $p$.

To show $i_{*}$ is injective we consider a loop $L$ that bounds a surface in $\hat{N}_{i}$. By Lemma $2.2, M$ is 1-acyclic at infinity. Thus, there is an $N_{j}$ so that every loop in $N_{j}$ bounds in $N_{i}$. Since $L$ is compact, $N_{j}$ can also be chosen to miss $L$. The surface bounded by $L$ can be cut off on $\mathrm{Bd} N_{j}$ and replaced by a surface completely contained in $N_{i}$.

Step 8 . The space $\hat{M}$ is a generalized 3-manifold. The local homology properties follow from the information filled in the long exact sequence. Also a finite dimensional, locally contractible, separable metric space is an ANR and in fact a retract of an open subset of a finite dimensional Euclidean space (see [4, p. 122]).

Step 9. The 3-manifold $M$ embeds in no compact 3-manifold. We use a result of [23] that is based on Haken's finiteness theorem for incompressible surfaces [9, §4]. Since each $Q_{i}$ has incompressible boundary, [23] says that if $M$ embeds in a compact 3-manifold $X$, then all but a finite number of $\mathrm{Cl}\left(X-K_{i}\right)$ would be solid tori. Since $X-M$ would be cell-like (see introduction), the map from a solid torus $\mathrm{Cl}\left(X-K_{i}\right)$ to $\hat{N}_{i}$ gotten by mapping $X-M$ to $p$ would be an isomorphism on $\pi_{1}$ [12]. Thus, a meridian for $\mathrm{Cl}\left(X-K_{i}\right)$ would go to a curve on $\mathrm{Bd} \hat{N}_{i}$ trivial in $\hat{N}_{i}$. Such a curve must be $X_{i}$. Since all but a finite number of the $\mathrm{Cl}\left(X-K_{i}\right)$ are solid tori, we can find an $i$ and $i+1$ where this holds.

Thus, $\mathrm{Cl}\left(X-K_{i+1}\right)$ is a solid torus with meridian $X_{i+1}$. But $X_{i+1}$ is $Z_{i}$, and $Q_{i}$ plus a solid torus with meridian sewn to $Z_{i}$ is $P-T_{1}$. This would say that $P-T_{1}$ is homeomorphic to $\mathrm{Cl}\left(X-K_{i}\right)$, a solid torus, with $X_{i}$ as a meridian. But this would imply $P \cong S^{3}$, contradicting our choice of $P$.

This proves Theorem 3 .

\section{REFERENCES}

1. S. Armentrout, Cellular decompositions of 3-manifolds that yield 3-manifolds, Mem. Amer. Math. Soc. no. 107 (1971).

2. R. H. Bing, Approximating surfaces with polyhedral ones, Ann. of Math. (2) 65 (1957), 456-483.

3. N Necessary and sufficient conditions that a 3-manifold be $S^{3}$, Ann. of Math. (2) 68 (1958), $17-37$.

4. K. Borsuk, Theory of retracts, Monografie Mat., Tom. 44, PWN, Warsaw, 1967.

5. M. G. Brin, Improving 3-manifold compactifications of open 3-manifolds, Houston J. Math. 4 (1978), 149-163.

6. J. W. Cannon, The recognition problem: What is a topological manifold?, Bull. Amer. Math. Soc. 84 (1978), 832-867.

7. J. W. Cannon, J. L. Bryant and R. C. Lacher, The structure of generalized manifolds having nonmanifold set of trivial dimension, Geometric Topology (Proc. 1977 Georgia Topology Conf.), edited by J. C. Cantrell, Academic Press, New York, 1979, pp. 261-303.

8. C. H. Edwards, Jr., Open 3-manifolds which are simply connected at infinity, Proc. Amer. Math. Soc. 14 (1963), 391-395.

9. W. Haken, Some results on surfaces in 3-manifolds, Studies in Modern Topology, Math. Assoc. Amer., distributed by Prentice-Hall, Englewood Cliffs, N. J., 1968, pp. 39-98.

10. J. M. Kister and D. R. McMillan, Jr., Locally Euclidean factors of $E^{4}$ which cannot be embedded in $E^{3}$, Ann. of Math. (2) 76 (1962), 541-546.

11. T. Knoblauch, Imbedding deleted 3-manifold neighborhoods in $E^{3}$, Illinois J. Math. 18 (1974), $598-601$

12. R. C. Lacher, Cell-like mappings. I, Pacific J. Math. 30 (1969), 717-731.

13. __ Cell-like mappings and their generalizations, Bull. Amer. Math. Soc. 83 (1977), 495-553. 
14. D. R. McMillan, Jr., Some contractible open 3-manifolds, Trans. Amer. Math. Soc. 102 (1962), 373-382.

15. __ Strong homotopy equivalence of 3-manifolds, Bull. Amer. Math. Soc. 73 (1967), 718-722.

16. __ Acyclicity in three-manifolds, Bull. Amer. Math. Soc. 76 (1970), 942-964.

17. C. D. Papakyriakopoulos, On Dehn's lemma and the asphericity of knots, Ann. of Math. (2) 66 (1957), 1-26.

18. F. Quinn, Resolutions of homology manifolds, Notices Amer. Math. Soc. 26 (1979), A-130, Abstract \# 763-57-9.

19. F. Raymond, R. L. Wilder's work on generalized manifolds-an appreciation, Algebraic and Geometric Topology (K. C. Millett, Ed.), Lecture Notes in Math., vol. 664, Springer-Verlag, Berlin and New York, 1978, pp. 7-32.

20. R. Sternfeld, A contractible open $n$-manifold that embeds in no compact $n$-manifold, Ph.D. Thesis, Univ. Wisconsin, Madison, 1977.

21. J. Vrabec, Submanifolds of acyclic 3-manifolds, Pacific J. Math. 49 (1973), 243-263.

22. F. Waldhausen, Irreducible 3-manifolds which are sufficiently large, Ann. of Math. (2) 87 (1968), 56-88.

23. A. Wright and R. Messer, Embedding open 3-manifolds in compact 3-manifolds, Pacific J. Math. 82 (1979), 163-177.

Department of Mathematics, State University of New York at Binghamton, Binghamton, NEW YORK 13901 